\title{
Les sciences du territoire
}

Le territoire est l'un des attributs du pouvoir. Les galeries des cartes aménagées dans les palais de la Renaissance rappellent d'ailleurs avec force combien la représentation du territoire contribue à asseoir la puissance du prince. Toutefois, audelà de ces figurations allégoriques du contrôle exercé par le prince sur " son » territoire ou sur les savoirs géographiques de l'Ancien et du Nouveau Monde, la centralisation de données relatives au territoire apparaît, dès le XVIIe siècle, comme un recours possible pour donner au roi une image de son royaume. La connaissance de celui-ci renvoie à la tradition médiévale du miroir du prince, un traité destiné à instruire le prince chrétien de sa fonction et peindre ses vertus idéales. Lorsqu'à la Renaissance le pouvoir monarchique s'affirme, ce portrait moral est complété par une apologie de la puissance temporelle du souverain, d'où la nécessité de produire un tableau descriptif et général par lequel le prince pourra connaître l'état du royaume, d'où aussi, à partir de là, l'indispensable mise en œuvre d'enquêtes générales censées rassembler la matière d'un tel tableau. À mesure que l'organisation de l'État se structure et que le périmètre de l'action publique se précise, la connaissance des territoires mobilise savants et administrateurs qui déploient de concert des procédures d'enquête et mettent au point des outils spécifiques pour en exploiter les données (Bourguet, 1989). Les gestes de collecte et les tentatives d'ordonner les informations qui en résultent contribuent à façonner des sciences du territoire qui ne s'individualisent cependant qu'à la toute fin de la période moderne.

\section{L'enquête et la construction d'un savoir empirique sur le territoire}

Dès 1630, en France, le surintendant des finances adresse une circulaire aux intendants pour les prier de fournir des états descriptifs de leurs circonscriptions. En 1663 et 1664, Colbert rédige une instruction aux maîtres des requêtes qui encadre une grande série d'enquêtes générales. Par ce texte, il charge chacun d'eux d'organiser une enquête pendant quatre ou cinq mois dans une partie précise du territoire. L'objectif est d'obtenir une connaissance complète du territoire en 7 ou 8 années. Colbert élargit ainsi les compétences des intendants, il les conduit à veiller sur les capacités des contribuables, à s'occuper des questions économiques, à contrôler les communautés d'habitants. Cependant, seules huit provinces répondent à cette enquête.

En 1665, le maître des requêtes Le Peletier de la Houssaye propose d'élaborer un rapport susceptible de " donner au roy et à ses ministres une très entière et très parfaite connaissance de tous les lieux du royaume et de chacun en particulier ». Cette initiative conduit à la rédaction de plusieurs rapports. Toutefois, l'entreprise la plus connue en la matière reste celle des mémoires des intendants pour l'instruction du duc de Bourgogne. L'objectif qui préside à la réalisation de cette enquête tient à l'éducation du fils du Grand Dauphin, né en 1682. Il s'agit de produire un tableau aussi exact que possible du royaume qu'il devait gouverner. Le questionnaire mis au point par le duc de Beauvillier est adressé aux intendants en février 1697. Il est présenté comme universel et devant être ajusté aux ressources de chaque généralité. Il aborde 19 ensembles de questions, structurées d'une manière devenue classique dans les mémoires de statistique 
descriptive: géographie physique, ressources, démographie, rappel du maillage administratif, société et économie, évolution. D’après le questionnaire, les mémoires réalisés doivent à la fois produire un reflet de la société d'Ancien Régime et répondre aux préoccupations fiscales et mercantilistes du pouvoir royal.

$\mathrm{Au}$ même moment, le souci de la prospérité économique du royaume conduit à l'émergence d'une autre forme d'enquête, à finalité plus immédiate et pratique que pédagogique. Ce mouvement accompagne l'activité croissante des bureaux de la monarchie chargés de dénombrer la population, de faire l'inventaire des subsistances, d'enregistrer les prix, etc. En 1694, Pontchartrain ordonne un recensement complet de la population afin d'établir l'assiette de la première capitation. Procéder à de vastes entreprises de comptage, voire de calcul était déjà une pratique commune au XVIIe siècle. Les travaux de John Graunt et William Petty sur la population en témoignent. À partir des bulletins de mortalité disponibles depuis le début du XVIIe siècle, Graunt cherche à tirer des données quantitatives sur l'effectif et le mouvement de la population. Il publie en 1662 Natural and Political Observations... upon the Bills of Mortality. Ces travaux sont peu progressivement associés à un nouveau domaine de savoir "l'arithmétique politique », expression proposée par Petty dans le titre de l'un de ses ouvrages - Political Arithmetic - publié en 1690. Gregory King et Charles Davenant poursuivent cette manière d'aborder l'économie par les chiffres (Hecht, 1977).

$\mathrm{Au}$ cours du XVIIIe siècle, en France notamment, une pratique statistique plus régulière se met en place, indépendamment des préoccupations fiscales et des objectifs concrets immédiats. Ainsi, dans les bureaux des subdélégués et des intendants, l'habitude est-elle prise d'enregistrer le prix des produits agricoles et industriels pratiqués sur les marchés et de dresser, deux fois par mois d'abord, puis chaque semaine ensuite des " états » de subdélégation et de généralité. Ces documents sont transmis à Paris et permettent au bureau des subsistances de présenter au contrôleur général un «tableau général du royaume ». Des pratiques comptables régulières se mettent donc en place progressivement mais elles ne concernent que des domaines limités. À partir des années 1720, la collecte d'informations ne vise plus seulement à prélever les impôts mais à analyser l'évolution de la société et à faire de la statistique un moyen de prévision et d'action. Les enquêtes des contrôleurs généraux qui, au cours du XVIIIe siècle, cherchent à produire un état économique du pays en témoignent. Un nombre de plus en plus important de questions appelle des réponses quantifiées. Ainsi, dans l'enquête lancée par Orry en 1730, le Contrôle général demande aux intendants d'indiquer non seulement le nombre d'habitants, mais aussi les prix des matières premières, de la main d'œuvre, le nombre d'ateliers, les productions, etc. À ces enquêtes générales, viennent s'ajouter au cours du XVIIIe siècle, des enquêtes particulières sur les mines (en 1741, 1742,1764 et 1783), sur la métallurgie (en 1772, 1774, 1788), sur les tanneries (en $1733,1745,1759,1788)$. Bien que ces enquêtes mêlent des éléments descriptifs avec des données quantitatives, elles témoignent à partir des années 1740 de la formalisation d'une science pratique du dénombrement industriel, conçue sur un modèle assez proche de celui de la statistique démographique mis au point par les arithméticiens.

L'intérêt que suscitent ces sciences du territoire naissantes au milieu du XVIIIe siècle contribue à multiplier les enquêtes au-delà de la sphère de l'administration (Brian, 1994). Si certains serviteurs de l'État élaborent des questionnaires censés formaliser 
l'enregistrement des données, ils les testent dans le cadre d'initiatives individuelles. Dès la fin du XVIIe siècle, Vauban conçoit plusieurs documents de ce type dont un agenda intitulé «pour faire l'instruction du dénombrement des peuples et la description des pays ». Il met lui-même à l'épreuve cette liste de questions en l'appliquant à l'élection de Vezelay (Virol, 2003). Dans les années 1770, Munier, qui est inspecteur des Ponts et Chaussées, compose un Recueil d'observations qu'il applique à l'Augoumois et dont il publie les résultats en 1779, déconnectant toutefois cette publication qui relève du champ de l'érudition de son activité professionnelle (Perrot, 1977). Au-delà de ces cas isolés, des synergies fortes apparaissent entre les états et certaines sociétés savantes. L'Enquête du Régent organisée depuis l'Académie royale des sciences en témoigne. [cf. focus Enquête du Régent]. À partir de 1754, en Angleterre, la Society for the Encouragement of Arts, Manufactures and Commerce apparaît comme un relai essentiel de l'action du Board of Trade (Hilaire-Pérez, 2000). De même qu'elle organise des prix censés encourager l'activité, cette société fait de l'enquête un moyen de développement économique.

D'autres dynamiques du même type émergent, en France, dans le domaine de la statistique médicale, criminelle ou démographique. Elles témoignent d'une volonté croissante chez certains savants d'accéder à la connaissance de la société en partant des difficultés vécues par la population. C'est bien cette préoccupation qui conduit le médecin rouennais, Lepecq de la Clôture à publier en 1778 sa Collection d'observations sur les maladies et constitutions épidémiques. L'ouvrage est présenté comme le résultat d'une quinzaine d'années d'observations qui donnent la matière des premières topographies médicales importantes du royaume de France. Pour rassembler les données de son étude, Lepecq de la Clôture crée un réseau de correspondants vivant essentiellement dans l'ouest de la France. La même année, Vicq d'Azyr expose à la Société royale de médecine un projet d'enquête assez similaire, à ceci près qu'il l'étend à l'échelle du pays entier (Mandressi, 2008). Outre qu'elles ont la particularité de mobiliser des réseaux savants ou des communautés professionnelles, ces enquêtes ne se contentent pas de dresser des constats, elles doivent établir des liens entre les phénomènes observés, expliquer l'origine des symptômes, proposer des diagnostics. De telles enquêtes contribuent aussi à élargir les connaissances de l'administration sur la société ainsi que le champ de l'action des gouvernements.

En 1784, dans son traité De l'administration des finances, Necker présente un projet d'institut de données censé collecter les renseignements auprès des administrations, les vérifier et publier un bilan tous les ans (Perrot, 1992). Aucune instance de ce type n'est jamais mise en place mais d'autres supports - de papier ceux-là - sont imaginés au XVIIIe siècle afin de dépasser le stade de l'accumulation et produire du sens à partir des données collectées (Denis, Lacour, 2016).

\section{Ordonner la connaissance des territoires : la constitution de nouveaux outils}

Que ce soit à Milan lorsqu'il est au service de Ludovic Sforza, ou à Florence au tout début du XVIe siècle, Léonard de Vinci recourt à des représentations figurées du territoire pour accompagner les propositions d'aménagements hydrauliques et les préconisations techniques qu'il adresse à ses mécènes. Si singulière que soit la 
biographie de Léonard, cet exemple montre que recourir à la carte pour administrer un territoire ne peut être regardé comme une invention du siècle des Lumières [cf. chapitre 8.3 Cartographie]. Cependant, au cours de la première moitié du XVIIIe siècle non seulement l'usage des cartes se généralise dans les bureaux de l'administration, mais les États européens prennent des initiatives qui contribuent à accroître le nombre des représentations cartographiques de leur territoire et à en généraliser l'usage. Que l'on songe aux cadastres commandés par les Habsbourg, à la carte de France confiée aux Cassini ou à la tradition des surveys (arpentages) très répandue en Angleterre dès le XVIe siècle, tous ces documents rendent les territoires visibles en un coup d'œil, ils concourent à l'identification de leurs ressources ainsi qu'à l'élaboration de projets d'exploitation censés les valoriser au mieux.

Dès la fin du XVIIe siècle, certains mémoires produits dans le cadre de l'enquête du duc de Beauvillier pour permettre au jeune duc de Bourgogne de découvrir la diversité des provinces françaises sont accompagnés de cartes de localisation (Virol, 2003). En janvier 1735, Philibert Orry, Contrôleur général, adresse à tous les intendants une lettre circulaire dans laquelle il expose les projets cartographiques que l'administration royale souhaite mettre en œuvre. Il explique combien dresser des cartes de France s'avère indispensable à l'essor du commerce et de la navigation intérieure. Ce texte assigne aux ingénieurs des Ponts et Chaussées un rôle déterminant dans l'identification des ressources économiques du territoire et l'amélioration des conditions de leur circulation. Deux années plus tard, alors que la monarchie vient d'instaurer la corvée afin d'améliorer l'état des chemins, les ingénieurs des Ponts et Chaussées sont mobilisés de manière beaucoup plus impérative pour produire les cartes qui doivent accompagner les travaux routiers. Ils sont tenus de lever un plan assorti d'un mémoire descriptif et, le cas échéant, de profils pour le suivi de chaque chantier. Une échelle commune est arrêtée pour tous ces documents censés donner à voir les travaux d'amélioration du réseau routier principal : élargissements des voies, rectifications, adoucissements de pente, plantations d'alignement, etc. Ces planches composent les atlas des routes de France, communément nommés "Atlas de Trudaine». Sur les 3000 planches manuscrites élaborées entre 1745 et 1780 par les ingénieurs des Ponts et Chaussées, les routes faites ou à faire sont représentées et resituées dans leur environnement immédiat (Blond, 2014).

À la même période, certains membres du corps utilisent l'outil cartographique pour accompagner la mise en œuvre d'un inventaire, à l'image de ce Projet d'une carte de France (...) destinée pour l'administration des Ponts et Chaussées qui propose de cartographier sur un même fond les villes, les routes, les canaux et les fleuves, la situation des mines et fonderies, les détails des montagnes ainsi que le bilan des importations et exportations de chaque province du royaume. Cette double manière d'aborder l'acte cartographique - pour faciliter la compréhension d'un contexte local et produire une représentation synthétique de l'activité économique - se retrouve dans les usages des cartes mobilisées par l'administration des Mines.

À partir des années 1740, au moment où émergent des expressions comme « la science des finances », la «science du commerce» ou la " science de la guerre », l'usage de la «science des mines » se répand dans la langue française. Cette expression témoigne d'une préoccupation pour les ressources minérales de plus en plus marquée au sein de 
l'administration française. En effet, depuis l'arrivée de Philibert Orry au Contrôle général en 1730, ses bureaux s'informent des voies envisageables pour doter l'État de moyens de contrôle et surtout pour organiser une meilleure exploitation des richesses. De nouvelles méthodes de travail font alors leur apparition. A partir de 1741, les intendants sont chargés de l'administration des mines. Dans le même temps, le chimiste Jean Hellot, qui est essayeur à la Monnaie, se voit confier l'examen technique des projets relatifs à l'industrie et aux mines. Enfin, des inspections sont organisées, plus particulièrement dans les mines de charbon. On voit progressivement se constituer un savoir spécifique au sein du groupe professionnel mis en place de façon informelle par le Contrôle général, plusieurs décennies avant que le corps des Mines ne soit structuré. Au même moment, de nouvelles techniques de prospection et d'extraction apparaissent; elles suscitent partout en Europe une réflexion sur la nécessaire formation des exploitants, des techniciens et des mineurs. Dans cette même perspective de valorisation des ressources minérales, le géographe Jean-Louis Dupain-Triel bénéficie, au début des années 1780, d'un soutien du contrôle général pour poursuivre son projet de cartographie des ressources minérales. Une réduction de la carte de Cassini doit lui servir de fond de carte. L'instruction qu'il reçoit alors précise :

\footnotetext{
« Ces cartes offriront par provinces, les villes, les bourgs, les rivières, les grands chemins, les contours des forêts et des bois avec les lieux des établissements de verreries, fourneaux et autres usines et le point central des concessions. Ces cartes seront d'abord faites à la main. On attendra pour les faire graver que les renseignements puissent y permettre les corrections nécessaires. Il sera donc fait trois copies de chacune de ces cartes : l'une pour rester au dépôt des papiers du commerce ; une autre pour être remise à celui de MM. les intendants du commerce, dans le département duquel se trouve la généralité, et la troisième pour servir à MM. les inspecteurs des Mines à l'effet d'y marquer ce qu'ils auront observé dans leurs voyages ».
}

Ici, la carte n'est pas seulement un outil qui permet de figurer le territoire et ses ressources, elle constitue un recours pour organiser les éléments tirés des enquêtes (Laboulais, 2012).

Dans la seconde moitié du XVIIIe siècle, la carte est investie d'une nouvelle manière par les administrateurs, elle passe du statut d'image à celui d'outil. Le plus souvent, les administrateurs utilisent des cartes issues de relevés topographiques ou de travaux d'arpentage comme support pour consigner leurs observations. Ces cartes imprimées ou manuscrites leur permettent d'inscrire dans l'espace de leurs circonscriptions les observations contenues dans les mémoires ou, plus encore, dans les tableaux adressés à l'administration centrale. C'est de cette manière qu'apparaissent les premières cartes thématiques. Peu inventives du point de vue graphique, elles suscitent une simple translation des renseignements contenus dans les mémoires et les tableaux vers l'espace de la carte. Ces fonctions nouvelles assignées aux cartes constituent l'une des expressions de la territorialisation des savoirs administratifs caractéristique de la deuxième moitié du XVIIIe siècle (Margairaz, 2008). Conçues comme un outil capable de figurer les données tirées des enquêtes, les cartes deviennent de puissants outils mis au service de l'action.

Les sciences du territoire sont diverses dans leurs dénominations : l'économie politique, la statistique, la démographie, le caméralisme, l'arithmétique politique sont les sciences les plus immédiatement associées à l'action de l'État. On peut y ajouter la 
géographie et la minéralogie. Cependant, au-delà de cette diversité, elles présentent des similitudes dans leurs pratiques, témoignent de l'essor d'une forme de rationalité politique qui se déploie au cours du XVIIe siècle et prend une forme plus aboutie au XVIIIe siècle. Elles se caractérisent par la volonté de produire, de susciter, d'organiser le territoire afin de lui permettre de développer toutes ses propriétés.

\section{Références bibliographiques}

Bourguet, Marie-Noëlle. 1989. Déchiffrer la France : la statistique départementale à l'époque napoléonienne. Paris, Éd. des Archives contemporaines.

Blond, Stéphane. 2014. L'atlas de Trudaine. Pouvoirs, cartes et savoirs techniques au siècle des Lumières. Paris, CTHS.

Brian, Éric. 1994. La mesure de l'État. Administrateurs et géomètres au XVIIIe siècle. Paris, Albin Michel.

Hecht, Jacqueline. 1977. "L'idée de dénombrement jusqu'à la Révolution », dans Pour une histoire de la statistique, t. 1. Paris, INSEE, p. 21-81.

Hilaire-Perez, Liliane. 2000. L'invention technique au siècle des Lumières. Paris, Albin Michel.

Laboulais, Isabelle. 2012. «Aux origines de la carte géologique de France : retour sur les productions cartographiques du corps des Mines au cours du premier XIXe siècle », dans B. Belhoste, A.-F. Garçon (dir.), Les ingénieurs des Mines. Cultures, pouvoirs, pratiques, Paris, Comité pour l'histoire économique et financière de la France, p. 19-31.

Lacour, Pierre-Yves, Denis, Vincent, 2016. "La logistique des savoirs. Surabondance d'informations et technologies de papier au XVIIIe siècle », Genèses, 1, p. 107-122.

Mandressi, Rafael, 2008. « Le passé, l'enseignement, la science : Félix Vicq d'Azyr et l'histoire de la médecine au XVIIIe siècle ", Medicina nei secoli : Arte e Scienza. 20, Istituto di Storia della Medicina, Università degli Studi di Roma La Sapienza, p. 183-212.

Margairaz, Dominique. 2008. «Introduction. De Colbert à la statistique générale de la France », dans D. Margairaz \& P. Minard (dir.), L'information économique XVIe-XIXe siècle, Journées d'études du 21 juin 2004 et du 25 avril 2006. Paris, Comité pour l'histoire économique et financière de la France, pp. 143-153.

Minard, Philippe. 1998. La fortune du colbertisme. État et industrie dans la France des Lumières. Paris, Fayard, pp. 183-203.

Perrot, Jean-Claude. 1977. L'âge d'or de la statistique régionale française (an IV 1804). Paris, Société des études robespierristes. Vallon.

Virol, Michèle. 2003. Vauban. De la gloire du roi au service de l'État. Seyssel, Champ

Isabelle Laboulais, « Les sciences du territoire », dans L. Hilaire-Pérez, Fabien Simon, Marie Thébaud-Sorger, L'Europe des sciences et des techniques. Un dialogue des savoirs XVIe-XVIIIe siècle, Presses universitaires de Rennes, p. 399414. 